\title{
Correspondencia previa entre el Licenciado Don Vicente Díaz Samayoa, secretario de la Universidad de San Carlos de Guatemala, y las universidades convocantes al "I Congreso de Universidades Hispano-Americanas"
}

Este 2019, nuestra Unión de Universidades de América Latina y el Caribe, UDUAL, cumple 70 años. Fue en 1949, entre los días 15 y 22 de septiembre que se creó este lazo entre las universidades de una Hispano-América con un contexto muy distinto, pero al mismo tiempo tan difícil para nuestra educación superior como hoy en día. La Guerra Fría ataviaba al mundo y Nuestra América sentía los coletazos de ese enfrentamiento, mientras que se definían políticas disímiles y peligrosas.

Pese a este entorno, se decide hacer un "I Congreso de Universidades HispanoAmericanas", con sede en la Universidad de San Carlos de Guatemala, a la cual quedan invitados los institutos de educación superior de América Latina y el Caribe, para articular -como lo hemos hecho hasta la actualidad- nuestra solidaridad, fuerzas y conocimiento en pos del bienestar y la mejora de nuestras casas de estudio.

Agradecemos a Ingrid S. de Genovez, Documentalista, a Karen Barrios, Archivista, y a la Mtra. Amanda López, jefa del Archivo General de la Universidad de San Carlos de Guatemala (AGUSAC), por el envío de estos documentos tan preciados para la historia y el porvenir de nuestra UDUAL. Por eso, ahora contamos en el Archivo General de la UDUAL con esta correspondencia escrita por el secretario de la Universidad de San Carlos de Guatemala, Licenciado Vicente Díaz Samayoa, en respuesta a las universidades concurrentes, con anterioridad a la fecha puesta para el magno acontecimiento de nuestro primer encuentro.

Por una de estas cartas (fechada el 22 de enero de 1949), nos enteramos de que la Universidad Autónoma de México será una de las asistentes, y que también el temario definitivo del Congreso estará a cargo del Doctor Luis Alberto Sánchez, rector de la Universidad de San Marcos, en Lima, Perú, y organizador del evento. Asimismo, aparece entre estas epístolas (fechada el 29 de julio de 1949) la evocación del texto en el cual es mencionado el Congreso, "Misión de la Universidad en nuestra América", de Andrés Townsend Ezcurra, peruano, exiliado en Panamá; en ese agosto de 1949 es invitado a vivir en Guatemala por el presidente Juan José Arévalo,

POR ANALHI AGUIRRE Doctora en Teoría Literaria, especialización en género, espacio y psicoanálisis. UDUAL. analhi.aguirre@udual.org 
donde será profesor de la cátedra de Historia Moderna y Contemporánea en la Universidad de San Carlos de Guatemala. De igual manera, se presentan dos cartas (ambas fechadas el 25 de febrero de 1949), una al Ministro de Brasil, Dr. Carlos de Silveira Martin Ramos, y otra, al Embajador de Brasil, donde se les expresa la preeminencia de la representación de las universidades de Brasil en el programa, ya que: "El objeto del I Congreso de Universidades Latino-Americanas, [sic.] es tan importante y hemos recibido muy numerosas adhesiones que no dudamos del interés que las Universidades brasileñas, experimentarán con respecto a él". ${ }^{1}$

Con este preámbulo de entusiasmo, antecedente a la fundación de la UDUAL, compartimos la introducción de estos documentos, accesibles en nuestro repositorio, como otros tantos, donde se muestran no sólo las dificultades por las que han pasado nuestras universidades, sino también sus tinos.

\section{Nota}

1. Serie: expediente sobre el Congreso Latinoamericano de Universidades (Fundación UDUAL UNIVERSIDAD DE SAN CARLOS DE GUATEMALA), Archivo General de la Secretaría General de la UDUAL. 
Guatemala, 22 de enero de 1949.-

Sefior D1rector:

Ye es muy grato acusar recibo de su atento of1e1o de fecha 17 de enero de este afo,-

Ye permito 1 nforate que Ia atenta nota en que el'Sr. Rector de esa 1lustre Universidad comunicó a esta Rectorla que la institución a su digno cargo tomaria par te en el próximo Congreso de Universidades Fispano-Americanas, llegó a ésta en período de vacaclones; y por ello no fué contestada oportunamente.-

Con respecto a 103 otros dos puntos de su atenta nota, relativos al temario definitivo del Congreso y a otros datos concernientes al mismo, me perm1to manifestarle, que el Doctor don Luis Alberto Sánchez le proporcionará direg tamente la Informacion del casō, por ser él quien está encargado de 1e organización de dicha Convención Universitar1a.-

Al expresar a Ud. $1 \mathrm{~s}$ agradecimientos por su atención, aprovecho la oportunidad para suscribirme como su atento y seguro servidor.-

"ID Y BNSEINAD A TODOS" 
cutrMALA, C. A.

$$
\text { Guatemala, Ju1io } 29 \text { de } 1949 .
$$

Muy estimado Profesor:

We es honroso expresarle mls más cumplidos agradecimientos por $e 1$ envio de su interesante artfcu10 sobre la "Misión de la Universidad en nuestra América", en el cual hace referencla muy amable al Congreso Latino-America no de Universidades.-

$$
\text { Recibolo como un homenaje a nuestra- }
$$
casa de estudios, y esperamos segulr contando con su colabora ción para divulgar en la prensa panameria las noticias del 1 mportante Congreso Universitario.-

De usted con toda consideraclón y apre clo, afectislmo y seguro servidor.-

"ID Y ENSENAD A TODOS".

Señor Andrés Townsend Ezcurra, Ápartado 1576,

Pananá. Rep. de Panamá. 
ZSIDAD DE SAN CARLOS

DE GUATEMALA

guatrous, C. A.

Guatemala, 25 de eebrero de 1949.

Sefกor M1n1stro:

$\mathrm{Ba}$ sumamente grato para m1 comun1ear a Ud, que, de acuerdo con $1 \mathrm{~s}$ s autro $100708 \mathrm{y}-$ ue se reu 0, se de $=$ L canas.-

So ha adopatado eata resolue1́́n tomando muy ispecialmente en cuenta la ineludible necosidad de que lasUn1versidades del Bras1l, repábl1ca de que es Ud. digno representante, esté representadas en dícho certamen con toda amplitud, y que este carezca de toda restrico1ón.-

B1 objeto del I Congreso de Un1veraldades Lat1no-Amor1cnss, es tan 1mportante $\mathrm{y}$ hemos rec1b1do muy nu merosas adhesiones que no dudeanos del 1nterfs que las U n1versidades brasilefias experimenterán con respecto \& él, y pe permito solio1tar, por tento, encseseidamente a V.B. su val1osa cooperací́n una de cuyas fuentes, os sin duda, trasmitir, s1 lo tiene a blen, esta resolución de las U niversidades, tomada con el objeto de que las Universidades bresilefies, se sientan, como tienen derecho, plenamen to on su casa durante $10 \mathrm{~s}$ debetes del Congreso.-

Aprovecho le oportun1dad para relterar a Ud. Ia seguridad de m1 alta $y$ diatingulda consideración.-

"ID Y BUSERAD A TODOS"A.

Seflor Miniatro

de 1 Brsa11,

Dr. Carlos De S1lve1ra Martin Remos,

Presento. 